\title{
Dyslipidemia is not the only Risk Factor for AMI in Non-Diabetic Population: A Hospital Based Prospective Observational Study
}

\author{
R. Rajkakati ${ }^{1}$, S.M Baruah ${ }^{2}$, D. Timung ${ }^{3}$ \\ ${ }^{1}$ Associate Professor, Biochemistry ${ }^{2}$ Assistant Professor, Medicine ${ }^{3}$ PG Trainee, Medicine \\ Assam Medical College \& Hospital, Dibrugarh, Assam, India
}

\section{Background}

Myocardial infarction (MI) is the important manifestation of coronary heart disease. MI is myocardial necrosis occurring as a result of critical imbalance between coronary blood supply and myocardial demand. Myocardial infarction is the "impairment of heart function due to inadequate blood flow to the heart compared to its need, caused by obstructive changes in the coronary circulation to the heart". Acute myocardial infarction (AMI) is associated with obstruction of coronary artery, myocardial ischemia leading to myocardial necrosis and generation of reactive oxygen species (ROS). ${ }^{1}$ Diabetes mellitus (DM) increases the incidence of cardiovascular diseases (CVDs) and increases the risk of CVD-induced mortality in diabetic subjects compared to non-diabetic subjects. ${ }^{2,3}$ In more than $90 \%$ of cases, the cause of myocardial ischemia is reduced blood flow due to obstructive atherosclerotic plaque lesions in one of the three large coronary arteries or its branches. Coronary atherosclerosis is a complex inflammatory process characterized by accumulation of lipids, macrophages and smooth muscle cells resulting in the formation of intimae plaques in the large and medium sized epicardial coronary arteries. Dyslipidemia is one of the major risk factors for cardiovascular disease in diabetes mellitus. The characteristic features of diabetic dyslipidemia are a high plasma triglyceride concentration, low HDL cholesterol concentration and increased concentration of small dense LDL-cholesterol particles. The lipid changes associated with diabetes mellitus are attributed to increased free fatty acid flux secondary to insulin resistance. The increased risk of atherosclerosis in diabetes mellitus consists of multiple factors. Diabetesrelated changes in plasma lipid levels are among the key factors that are amenable to intervention. ${ }^{4,5,6}$

Although atherosclerosis occurs in the general population, some people are at greater risk of developing coronary artery disease. The etiopathogenesis leading to atherogenesis is still unknown. Epidemiological studies have identified several cardiac disease risk factors for MI. ${ }^{7}$ These risk factors can be classified as modifiable and non-modifiable. Modifiable risk factors are serum lipids, lipoproteins, Hypertension (HTN), Diabetes Mellitus (DM), smoking and tobacco chewing etc. Non modifiable risk factors are age, sex, genetics, and family history of CAD. Atherosclerotic changes due to dyslipidemia were more common in diabetic patients than non diabetics as per available previous researches.

The importance of the classical risk factors for heart disease was examined in the INTERHEART study, ${ }^{8}$ which is a large, international, standardized, case-control study from 262 centers in 52 countries from Asia, Europe, the Middle East, Africa, Australia, North America, and South America. All these classical and novel risk factors for cardiovascular disease would be expected to have varying relative contributions to the disease outcome in different populations. Although, thrombus formation is the proximate cause of acute myocardial infarction (AMI), atherosclerosis, the chief underlying cause, is a chronic progressive disease. ${ }^{9}$

The present study was conducted to assess the risk of myocardial infarction in non diabetic patients attending a Tertiary Care Hospital, Assam Medical College and Hospital, Dibrugarh, India.

\section{Aims and objectives}

1. To see the prevalence of dyslipidemia among Diabetic AMI

2. To see the prevalence of dyslipidemia among non-Diabetic AMI

3. To see the significance of dyslipidemia in these two groups

4. To see the other risk factors in non diabetic AMI like age, gender, positive family history, smoking, tobacco, hypertension and BMI.

\section{Material and Method:-}

This is a descriptive cross sectional study conducted in Assam Medical College and Hospital, Dibrugarh, Assam, India, during the period, Nov.2013 to Oct.2014. A total of 100 patients of MI were enrolled for the

Corresponding address: Dr. Dharamsing Timung, PGT, Department of Medicine, Assam Medical College \& Hospital, Dibrugarh, Assam, India. PIN: 786002 email: drdstimung86@gmail.com 
study. Out of which 50 patients of myocardial infarction were Non-Diabetic and remaining 50 were Diabetic. The diagnosis of MI was based on history of typical chest pain, ECG changes and rise in cardiac enzymes. All subjects were adult of age more than 30 years. Patients with suspected emboli of cardiac origin were excluded from the study. Selected patients were subjected to following protocol- 1. Detailed history 2. Detailed CVS examination 3. Blood sampling after 12 hrs. Fasting for [Serum total cholesterol, Serum triglycerides, Serum HDL, Serum VLDL, Serum LDL] 4. Blood sugar (FBS/PPBS) 5. Echocardiography 6. ECG 7. HbA1c 8. TropI.

The following risk factors were studied in these patients: (a)Family history of CAD: MI or sudden death before 55 years in father or $1^{\text {st }}$ degree male relatives or in mother before age of 65 years or other 1 st degree female relatives. (b) Smoking: currently smoking, or left smoking less than 3 months of the diagnosis. (c) Hypertension: Patients were considered hypertensive if already on antihypertensive therapy or reported to have blood pressure of more than $140 / 90 \mathrm{mmHg}$ on two or more occasions. (d)Fasting lipid profile: It was investigated within 48 hours of acute MI, because lipid profile is altered by acute MI, it tends to lower the HDL$\mathrm{C}$ and raises triglycerides. Total cholesterol $<200 \mathrm{mg} / \mathrm{dl}$, Triglycerides $<150 \mathrm{mg} / \mathrm{dl}$, LDL cholesterol $<100 \mathrm{mg} / \mathrm{dl}$ was considered normal. (e) Obesity: Patients with BMI $\geq 30 \mathrm{Kg} / \mathrm{m} 2$ were taken as obese and $<30 \mathrm{Kg} / \mathrm{m} 2$ as non obese. (f) Alcohol intake: The patients were inquired about the type and amount of alcohol they were using. Alcohol intake must not exceed 1-2 ounces of ethanol per day which is equivalent to 2-4 ounces of whisky/day, 8-16 ounces of wine per day, 24-48 ounces of beer/day. (g) OCP intake in females: The patients were asked about the current use of Oral contraceptive pills OCPs (currently using or left less than 3 months of the diagnosis of MI).

We divided the patients into two groups and the significance of each risk factor between the groups was calculated by employing the chi-square test, and $\mathrm{p}<0.05$ was taken as significant. The percentage, frequency and relative frequency of risk factors were also calculated.

\section{Results and observation.}

Table 1. Age and sex distribution of study cases

\begin{tabular}{|l|l|l|l|l|l|l|}
\hline AGE (in years) & \multirow{2}{*}{ No. of cases(n) } & Percentage(\%) & \multicolumn{2}{|c|}{ Male } & \multicolumn{2}{|c|}{ Female } \\
\cline { 4 - 7 } & & & N & $\%$ & N & $\%$ \\
\hline $31-40$ & 1 & 1 & 1 & 1.20 & 0 & 0.0 \\
\hline $41-50$ & 29 & 29 & 24 & 28.92 & 5 & 29.4 \\
\hline $51-60$ & 33 & 33 & 27 & 32.53 & 6 & 35.3 \\
\hline $61-70$ & 24 & 24 & 24 & 28.92 & 0 & 0.0 \\
\hline $71-80$ & 13 & 13 & 7 & 8.43 & 6 & 35.3 \\
\hline TOTAL & 100 & 100 & 83 & 100 & 17 & 100 \\
\hline
\end{tabular}

Table 2: Distribution of Diabetic and Non Diabetic Cases

\begin{tabular}{|l|l|l|l|l|}
\hline \multirow{2}{*}{ AGE GROUP(in years) } & \multicolumn{2}{|l|}{ DIABETIC } & NON-DIABETIC \\
\cline { 2 - 5 } & N & $\%$ & N & $\%$ \\
\hline $31-40$ & 0 & 0 & 1 & 2 \\
\hline $41-50$ & 17 & 34 & 12 & 24 \\
\hline $51-60$ & 12 & 24 & 21 & 42 \\
\hline $61-70$ & 12 & 24 & 12 & 24 \\
\hline $71-80$ & 09 & 18 & 04 & 8 \\
\hline TOTAL & 50 & 100 & 50 & 100 \\
\hline
\end{tabular}

Table 3: Demographic and clinical profile of patients admitted with Acute myocardial infarction

\begin{tabular}{|c|c|c|c|}
\hline Parameters & Diabetic & Non Diabetic & P-Value \\
\hline \multicolumn{4}{|l|}{ Age } \\
\hline Mean \pm Sd & $58.68 \pm 10.94$ & $57.9 \pm 10.37$ & \\
\hline$>55 y$ & $19(38 \%)$ & $38(76 \%)$ & 0.04 \\
\hline \multicolumn{4}{|l|}{ Sex } \\
\hline Male & $41(82 \%)$ & $42(84 \%)$ & 1.00 \\
\hline Female & $9(18 \%)$ & $8(16 \%)$ & 1.00 \\
\hline \multicolumn{4}{|l|}{ Personal Habits } \\
\hline Smoking & $13(26 \%)$ & $32(54 \%)$ & 0.02 \\
\hline Alcohol & $13(26 \%)$ & $15(30 \%)$ & 0.83 \\
\hline Tobacco & $21(42 \%)$ & $23(46 \%)$ & 0.85 \\
\hline \multicolumn{4}{|l|}{ Occupation } \\
\hline Govt.Employee & 21 & 19 & \\
\hline Businessman & 17 & 15 & \\
\hline Farmer/Daily Wage Earner & 4 & 10 & \\
\hline Housewife & 8 & 6 & \\
\hline Lipid Profile & & & \\
\hline Chol & $173.4 \pm 41.3$ & $152.6 \pm 33.6$ & 0.006 \\
\hline
\end{tabular}


Dyslipidemia, Not The Only Risk Factor For Ami In Non-Diabetic Population: A....

\begin{tabular}{|c|l|l|l|}
\hline \multicolumn{1}{|c|}{ TGL } & $135.9 \pm 65.5$ & $116.7 \pm 36.4$ & 0.07 \\
HDL & $37.57 \pm 11.67$ & $35.95 \pm 11.48$ & 0.48 \\
LDL & $95.35 \pm 34.5$ & $89.2 \pm 29.9$ & 0.34 \\
Hypertenive & $31(62 \%)$ & $21(42 \%)$ & 0.30 \\
Non-Hypertensive & $19(38 \% 0$ & $29(58 \%)$ & 0.29 \\
Trop-I & $8.67 \pm 8.71$ & $7.38 \pm 7.26$ & \\
BMI & 5 & 3 & 0.21 \\
Obese(>30) & 45 & 49 & \\
Non-Obese(<30) & 9 & 23 & \\
Family History & 49 & 47 & \\
Outcome & 1 & 3 & \\
Improved & & \\
Expired & & \\
Duration of Hospital Stay & & \\
<7 Days & 2 & 3 & \\
>7 Days & 7 & 5 & \\
Taking OCP & & \\
Not Taking OCP & & \\
\hline
\end{tabular}

In the present study, total number of study subjects was 100 (50 non-diabetic and 50 diabetic MI cases). Out of which, $83 \%$ subjects were males and $17 \%$ were females. Male to Female ratio was found to be approximately 4.9:1. The median age was $58.29 \pm$ SD of 10.61 yrs. In non-diabetic group $76 \%$ cases were $>55 \mathrm{yrs}$ age, where as in diabetic group it occupies $38 \%$ cases $(\mathrm{p}<0.05)$. Approximately $32 \%$ of diabetic and $10 \%$ of non-diabetic patients were found to have Total cholesterol level of more than $200 \mathrm{mg} / \mathrm{dl}(\mathrm{p}<0.05)$. In this study, $32 \%$ of diabetic and $18 \%$ of non-diabetic patients had higher TG level, whereas $40 \%$ of diabetic and $26 \%$ of non-diabetic patients were found to have high LDL cholesterol level ( $p>0.05$ ). In the study, it was observed that $44 \%$ of diabetic and $54 \%$ of non-diabetic patients had HDL level less than $35 \mathrm{mg} / \mathrm{dl}$. Overall, Lipid profile level was found insignificant among the two groups. $26 \%$ diabetic and $54 \%$ of non-diabetic patients were smokers (p<0.05). $26 \%$ of diabetic and $30 \%$ of non-diabetic cases took alcohol whereas, $42 \%$ of diabetic and $46 \%$ of non-diabetic patients consumes tobacco ( $p>0.05$ ). Family history of premature coronary artery disease was present in $18 \%$ of diabetic and $46 \%$ of non-diabetic patients $(\mathrm{p}<0.05)$. Among the diabetic patients $62 \%$ cases and $42 \%$ cases of non-diabetic patients were hypertensive ( $p>0.05$ ), whereas, $10 \%$ of diabetic and $6 \%$ of non-diabetic were obese $(\mathrm{p}>0.05)$. Among the diabetic females $4 \%$ cases took OCPs where as $6 \%$ of nondiabetic females took OCPs $(\mathrm{p}>0.05)$. The comparison of risk factors in the two groups showed that higher age, smoking and family history of coronary disease had significant difference between the two groups. Other risk factors did not show significant difference between the two groups. The level of lipid profile in non-diabetic patients were not significant as compared with the diabetic patients.

There was no significant difference between the two groups regarding the outcome but the non-diabetic patients had lesser than 7 days duration of hospital stay as compared to the diabetic patients i.e $86 \%$ vs $18 \%(\mathrm{p}<0.05)$.

\section{Discussion:-}

Coronary artery disease remains a leading cause of death and exerts a heavy social and economical toll. ${ }^{10}$ The risk definitely increases with the increasing age. According to the results of this study, there is a linear relation between age and $\mathrm{MI}$ in the non-diabetic patients in the age group more than 55 years as compared to diabetic patients i.e $7 \%$ vs $38 \%(\mathrm{p}<0.05)$. Male sex is an important risk factor for CAD. The lifetime risk of $\mathrm{CAD}$ is one in three for women, and lifetime risk of developing CAD at 40 years of age is $50 \%$ for men and $33 \%$ for women. ${ }^{11}$ CAD is significantly low in premenopausal women because of oestrogen, but the diabetes takes away this advantage. ${ }^{11}$ In our study $83 \%$ of patients were male. This finding is consistent with the study of Akhtar et al ${ }^{12}$, who showed $85 \%$ of male patients in their study. The male gender showed a non significant difference between the two groups in our study. This is because most of the patients were male in both the groups, as male sex remains an important risk for IHD. Family history of CAD has an increased risk of premature coronary events. ${ }^{11}$ Our study showed that $46 \%$ of non-diabetic patients had family history of premature CAD as compared to $18 \%$ in diabetic patients, whereas Akhtar et al ${ }^{12}$ showed $57 \%$ of their patients to have positive family history of CAD.

Epidemiological studies suggest that patients with elevated lipid levels are at higher risk for atherothrombotic myocardial infarction than those with normal values and this applies mainly to premature ischemic cardiovascular disease. Although serum lipids have been incriminated in the pathogenesis of atherosclerosis, very little is known concerning the possible inter-relationship between abnormal levels of serum lipids and development of vascular complications. Belief in the possibility of such interrelationship arouse in part from the observation that lipid composition of the intima of the arteries resembles that of serum. Hence, Dyslipidemia has emerged clearly as a major risk factor associated with increased risk of atherosclerosis. The 
reason for increased prevalence of dyslipidemia is not known, however genetic factors and dietary habits seem to be important.

In our present study, there was a significant increase in Total Cholesterol in diabetic patients as compared to non-diabetic patients ( $32 \%$ vs $10 \%$ respectively) whereas the levels of other lipid profile did not show a significant differences in the two groups. TG brings significant change in LDL particle size, density, distribution and composition producing small dense LDL which is more atherogenic. In accordance to our study, Nigam found no significant correlation between LDL and risk of CHD. ${ }^{13}$

Smoking is the most important preventable cause of CAD. ${ }^{14}$ In our study $54 \%$ of non-diabetic patients were smokers compared to $26 \%$ in diabetic patients $(\mathrm{p}<0.05)$. Smoking causes premature CAD due to a number of factors. It has unfavourable effects on lipoprotein, it decreases HDL. ${ }^{15}$ Smoking also enhances platelet aggregability, promotes endothelial dysfunction, and causes coronary artery vasospasm. ${ }^{16}$ The studies of Pais et $\mathrm{al}^{17}$ conducted in India have shown very high percentage $(55 \%)$ of patients to be smokers. Hypertension is firmly established as a risk factor for IHD. In our study $42 \%$ of non-diabetic and $62 \%$ of diabetic patients were hypertensive. It is comparable to the study conducted by Akhtar et al in 1993 on young patients of IHD 47.6\% of their patients to be hypertensive. ${ }^{12}$ Obesity is an independent risk factor for CAD in both men and women. ${ }^{18}$ But in our study only $17 \%$ of patients were obese with a no significant difference between the groups. Alcohol and OCPs intake are not important risk factors in our society and our study proves it to be so.

Conclusion:-

Epidemiological studies suggest that patients with elevated lipid levels are at higher risk for atherothrombotic myocardial infarction than those with normal values. In the current study, no significant differences was found between the two groups with regards to serum lipid profile with risk of myocardial infarction. Age, male gender, family history of premature CAD and smoking were found to be more significant in the non-diabetic patients AMI. Hence, we would like to conclude that these factors are at more risk for AMI than dyslipidaemia in non-diabetic patients.

\section{References}

[1]. S. Kasap, A. Gonenç, D.E. Sener, I. Hisar.Serum cardiac markers in patients with acute myocardial infarction: oxidative stress, Creactive protein and N-terminal probrain natriuretic Peptide.J Clin Biochem Nutr, 41 (2007), pp. 50-57

[2]. C. Kalofoutis, C. Piperi, A. Kalofoutis, et al.Type II diabetes mellitus and cardiovascular risk factors: current therapeutic approaches.Exp Clin Cardiol, 12 (2007), pp. 17-28

[3]. D.M. Ansley, B. Wang.Oxidative stress and myocardial injury in the diabetic heart.J Pathol, 229 (2013), pp. 232-241

[4]. K. Park. Epidemiology of chronic non communicable diseases and conditions.In:Park"s Textbook of Preventive and Social Medicine. 20th ed. Jabalpur: M/S BanarasidasBhanot Publishers .2009:315-345.

[5]. Vinay Kumar, AbulK,NelsonFausto.The Heart .In:Robbins and Cotran"sTextbook of Pathological basis of Disease.9th edn;2009.Saunders. ElsevierPublishers, Philadelphia, Pennysylvania

[6]. Robert K Murray et al. Lipid transport and storage. Harper"s IllustratedBiochemistry. McGraw Hill Companies,28th edn; page 212223.

[7]. P.Beck, D.Bhawnani, N.Verma, D. Kumar et al.A Study on Lipid Profile in Non-DiabeticMyocardial Infarction Patients attending a Tertiary Care Hospital of Raipur City (C.G.), India. Indian Journal of Research.july, 2015; vol 4:issue 7

[8]. Yusuf S, Hawken S, Ounpuu S, Dans T, Avezum A, Lanas F, et al. INTERHEART Study Investigators. Effect of potentially modifiable risk factors associated with myocardial infarction in 52 countries (the INTERHEART study): Case-control study. Lancet. 2004;364:937-52.

[9]. Ridker PM, Cushman M, Stampfer MJ, Tracy RP, Hennekens CH. Inflammation, aspirin, and the risk of cardiovascular disease in apparently healthy men. N Engl J Med. 1997;336:973-9.

[10]. Ford ES, Capewell S. Coronary Heart Disease Mortality Among Young Adults in the US. From 1980 through 2002. J Am Coll Cardiol 2007;50:2128-32.

[11]. Llyod-Jones DM, Larson MG, Beiser A, Levy D. Lifetime risk of developing coronary artery disease. Lancet 1999;353:89-92.

[12]. Akhtar J, Islam N, Khan J, Risk factors and outcome of ischemic heart disease in young Pakistani adults. Specialist 1993;9:123-6.

[13]. P K Nigam, V S Narain and M Hasan.Serum lipid profile in patients with acute myocardial infarction. Ind J ClinBiochem; 2004; 19(1); 67-70.

[14]. Hennekens CH. Coronary disease: risk intervention. In: Julian DG, Wenger NK, (editors), Women and heart disease, 1st ed., London: Martin Dunitz; 1997. p. 39-48.

[15]. Cullen P, Schulte H, Assmann G. Smoking, lipoproteins and coronary heart disease risk. Data from the Munster Heart Study (PROCAM). Eur Heart J 1998:19:1632-41.

[16]. Sugiishi M, Takatsu F. Cigarette smoking is a major risk factor for coronary artery spasm. Circulation1993;87:76-9.

[17]. Pais P, Pogu J, Gerstein H, Zachariah E, Savitha D, Jayprakash S, et al. Risk factors for acute myocardial infarction in Indians: A case control study. Lancet 1996;348:358-63.

[18]. Willett WC, Manson JE, Stampfer MJ, Colditz GA, Rosner B, Speizer FE, et al. Weight, weight change, and coronary heart disease in women. Risk within the 'normal' weight range. J Am Med Assoc1995;273:461-5. 